\title{
Stochastic point process applied in aggregates composition for concrete
}

\author{
Cássia Vanessa Albuquerque de Melo ${ }^{1}$. Paulo César Correia Gomes ${ }^{1} \cdot$ Catarina Nogueira de Araújo Fernandes $^{2}$. \\ William Wagner Matos Lira²
}

Received: 18 September 2019 / Accepted: 1 November 2019 / Published online: 7 November 2019

(c) Springer Nature Switzerland AG 2019

\begin{abstract}
The objective of this work is to apply a stochastic point process technique in the evaluation of aggregate composition parameters for concrete. Several experimental, numeric and computational methods have been used for determining an ideal packing of aggregates for concrete, based on the principle of the lowest content of voids. Experimental method is quite hard-working. On the other hand, the mathematical and computational models need potent computer and be tested experimentally. Equipment and computational methods have been developed, to provide images of the particle distribution, approaching the actual distribution of aggregates and allowing to obtain ideal aggregates compositions. This work uses a stochastic point process, based on the point processes method, which a simple sequential inhibition (SSI) process on the arbitrary closed region places the particles. The SSI generates image of spherical particles distributions for viewing and checking compliance of parameters of aggregates compositions. The characteristics of aggregates (porosity, granulometry, proportion of each aggregate composition, and specific mass) and the problem's domain are input data. The SSI's output data of interest are virtual image of particles distribution, particles composition, porosity of each composition, diameter and number of particles. From SSI's output data, it can be determined the packing factor and a histogram of particles diameters. This information is evaluated and compared with the input composition. As a result, the stochastic SSI demonstrates to be efficient in comparing the output and experimental data, complying with the purpose of the study.
\end{abstract}

Keywords Stochastic point process technique $\cdot$ Aggregates composition $\cdot$ Packing factor $\cdot$ Concrete

\section{Introduction}

As a composite, concrete is constituted by two phases, one cement paste (fine particles with diameter less than $125 \mu \mathrm{m}$ ) and one another granular skeleton (aggregates with diameter higher than $125 \mu \mathrm{m}$ ) [1], it must have appropriate proportions of these phases that meet the required fresh and hardened concrete properties. In this context, it is important to study aggregate compositions, considering the influence of aggregates on the properties, such as workability, strength and deformability, and the significant portion that these materials occupy in the total volume of the concrete, around $70 \%$.

The more performance is required of the concrete, the better optimized aggregates composition should be. For this, different design mixture methods, aiming the aggregates packing, have been developed. The acquisition of ideal aggregate compositions, including their maximum compactness and the search for the minimum paste volume, has been the objective of several

$\checkmark$ William Wagner Matos Lira, william@lccv.ufal.br; Cássia Vanessa Albuquerque de Melo, cassiamelo@ctec.ufal.br; Paulo César Correia Gomes, pgomes@ctec.ufal.br; Catarina Nogueira de Araújo Fernandes, catarina@lccv.ufal.br | 1 Laboratory of Materials and Structure, Federal University of Alagoas, Maceió, Brazil. ${ }^{2}$ Laboratory of Scientific Computing and Visualization, Federal University of Alagoas, Maceió, Brazil. 
experimental, analytical and computational methods, by determining an ideal packing of aggregates for concrete, based on the principle of the lowest content of voids [2-4].

Some experimental and analytical methods, such as developed by Fuller-Thompson, Bolomey, Joisel and Faury [5], they have reference curves that allow to obtain an ideal granulometric curve of aggregate composition, which should represent a best continuing of particles in the granulometric fractions. In this way, the main aim of these methods is ensure a maximum possible compactness, minimizing the void content. Díaz [6] also presents a particle packing analysis through the optimum relation of the fine and coarse aggregate mixture, which can result in higher compactness and lower cement consume.

In the analytical scope, packing models for concrete have been proposed from optimized aggregates granulometric distribution $[7,8]$. These models are excellent tools for determining particle packing and are based on discrete approaches of particles and continuous distributions, being composed of grains of different sizes. These analytical models are classified in binary, ternary and multimodal mixtures [9].

Still in this analytical context, De Larrard [10] suggests a procedure for particle packing, namely Compressive Packing Models (CPM), which is an analytic model that considers the concept of both wall and loosing effect. The procedure is based on interaction among the coarse and fine grains of mixture to predict its packing. In a study by Fennis [11], the applicability of Compaction-Interaction Packing Model (CIPM) has been performed better in numerical simulation, mainly, because is capable of predicting the packing densities precisely considering the crushed aggregates. Beside both effects (wall and loosing effects), the CIPM can be interpreted as an improved model, resulting in minor error compared with experimental data. According to Larrard [10] and Fennis [11], the combination of a system of fractions of aggregates, which results in a lower porosity or higher density of packing, is crucial if it wanted to reduce the volume of voids and, consequently, the cement paste volume that fill the space between aggregate grains.

Few models can precisely estimate the grain packing density. The CPM and CIPM have stood out representing better the granular system, taking account different particles sizes to obtain smaller void content. Based on the theory that the void spaces between the larger particles may be filled by smaller particles, it is possible to reduce the volume of voids and, therefore, to increase of the packing density $[9,10]$. So, parameters such as diameter, shape and distribution of particles have been extensively investigated due to the impact that they have on the packing density [12] and in the concrete properties [13].
In this context, the use of numerical methods becomes a powerful tool for the analysis of these properties, mainly the Discrete Element Method (DEM) due to their characteristics related to efficient treatment of discontinuous and granular media. Among the problems where the method has been applied, it may cite soil and rock modeling, fragmentation, fracture, impact, and collision phenomena, in addition to those directly related to aggregate composition as part of studies of concrete problems [14-17]. HE et al. [12] affirm that the DEM has an appropriate approach to obtain the particle packing. Stroeven et al. [2] also used the DEM approach because it is economic, efficient and potentially reliable option for investigating the nonuniformities in the distribution of aggregates grains on a meso-level and so the cement paste on a micro-level. Generally, DEM is advantageous over the other methods because its theory is based on models of particle packing generated randomly on the assumption of spherical shape of the grain, with smooth surfaces and no attraction or repulsion of forces between them. For other hand, the use of spheres requires only a size as parameter (diameter).

One of the most relevant issue for calculating particle size is the overall spatial distribution of group of particles on a domain [18]. As in several areas of interest, particlesize distribution also is required for characterizing concrete. It is normally presented as the percentage of its total volume occupied by a given size fraction (aggregates). Due to the shape heterogeneity and density of the particles, computation of the particle-size distribution for aggregates is not a trivial task [19].

Basically, there are two types of procedures used to place particles on domains: dynamic and geometric algorithms. The most well-known shapes of these particles are disks and spheres, respectively applied to two- and threedimensional models.

Dynamic algorithms apply mechanical external forces to obtain an initial model of packed particles. An overview of classical dynamic techniques can be found in $[20,21]$. The use of these procedures generates a lot of particles homogeneously well distributed and in geostatic balance. In addition, porosity can be controlled by filling arbitrary domains with predefined irregular particle size. A disadvantage of these methods is its high computational cost. The geometric algorithms are well suited when, for example, one wants to gain computational speed.

Another category of procedures is based on purely geometric concepts. These geometric algorithms do the particle packing without DEM simulations. These techniques are strongly applied in the study of granular media, being widely found in the literature. Several techniques are available for the generation of randomly distributed disks or spheres with different diameters [21-24]. Normally these procedures are much faster than the dynamic 
ones because not need numerical simulations. However, traditional geometric algorithms generally can't provide a collection of particles placed on domain and meeting specified granulometric curves. These algorithms also may not guarantee geostatic balance of the particles and it presents some limitations on particle generating on geometrically complex domains.

In this context, Frery et al. [25] present a procedure for generating a particle set, granting its porosity and granulometric properties, in arbitrary closed domain. Its main application is the simulation of granular media described by disks and spheres. The spatial homogeneity is granted since it is considered that the voids are fully filled by cement paste and the particles are suspended, so the system is in global equilibrium. The particles are placed by a simple sequential inhibition (SSI) process on the domain. The SSI is a stochastic point process in which attraction or repulsion is not considered, the final state is static, particles arrive sequentially and there is no relaxation.

So, the objective of this work is to apply a stochastic point process technique in the analysis of aggregate compositions for concrete from parameters determined experimentally, where the characteristics of these compositions include distribution, porosity, sizes and number of particles. Disks are used to represent aggregates, and these are compared as results are achieved with experimental data, checking the potential of procedure as a numerical tool applied to technology of concrete.

The technique presented by Frery et al. [25] is used, adapting it to reproduce the study proposed in this work. An arbitrary number of radii sampling is obtained from a granulometric curve, and these particles are placed in an arbitrary closed region of the plane, using a stochastic point process. The number of particles to be used is iteratively determined in order to satisfy a predefined porosity.

The proposed strategy is comprised of the following steps:

1. Obtaining of experimental data.

2. Particle size generation:

(a) A granulometric curve and the porosity are used as input.

(b) An approximate number of particles that provide the desired porosity is calculated.

(c) The radii of the particles are samples of independent identically distributed random variables obeying the adopted granulometric curve.

(d) The particles are placed by a simple sequential inhibition (SSI) process on the arbitrary closed region. The number of particles is determined iteratively as to produce an approximation of the desired porosity.
3. Study of some parameters in the analysis of aggregate composition for suspended particle, which are represented by particles placed closed region. The parameters evaluated include the packing factor and frequency histogram of each composition.

The result of these steps is a plausible model of particle distribution used in analyses of aggregate composition for concrete, obtained with low computational cost due to its geometric nature. This procedure is able to simulate aggregate composition of low porosity, which are usually obtained by dynamical techniques that are much more time consuming. Such models can be used as input data for numerical simulations of aggregate composition for concrete using the discrete element method.

\section{Experimental data}

To apply the stochastic point process technique in the analysis of aggregate compositions for concrete proposed in this work, beyond of domain of the problem, the parameters determined experimentally necessary as input data are experimental porosity (ne), granulometry of aggregates, proportion of each aggregates composition, and specific mass of dry aggregates. In further to these input data, some additional information used in the numerical simulations are composition (proportion) of the aggregates and specific mass of dry aggregates. However, these parameters are not relevant in the point process technique used here.

In this work are used aggregates with two small grain aggregates: fine sand (F) and medium sand (M), and two large grain aggregates (G12) and (G19) of maximum dimensions equivalent to $12.5 \mathrm{~mm}$ and $19.0 \mathrm{~mm}$, respectively. The Fig. 1 illustrates the granulometric curve of the all solids materials, used in the aggregate composition, obtained according to NBR NM 248:2003 [26].

The aggregate compositions are determined through the highest unitary mass and the lowest voids content, according to NBR NM 45:2006 [27], method " $C$ " in loose state, without compaction.

Proportioning of aggregates for concrete is used to determine the optimal aggregate combination. The test procedure is as follows: (1) the aggregates proportions are determined, firstly, for a binary composition. The test starts with $100 \%$ of coarse aggregate. The test continues with putting fine or medium aggregate. These aggregate are mixed and poured into the container without any compaction; (2) the excess aggregates remaining above the top of the cylinder are struck off; (3) the proportions ranged of 10 in 10 percent of $100 \%$ until the coarse aggregate proportion of $60 \%$ and, after, of 5 in 5 


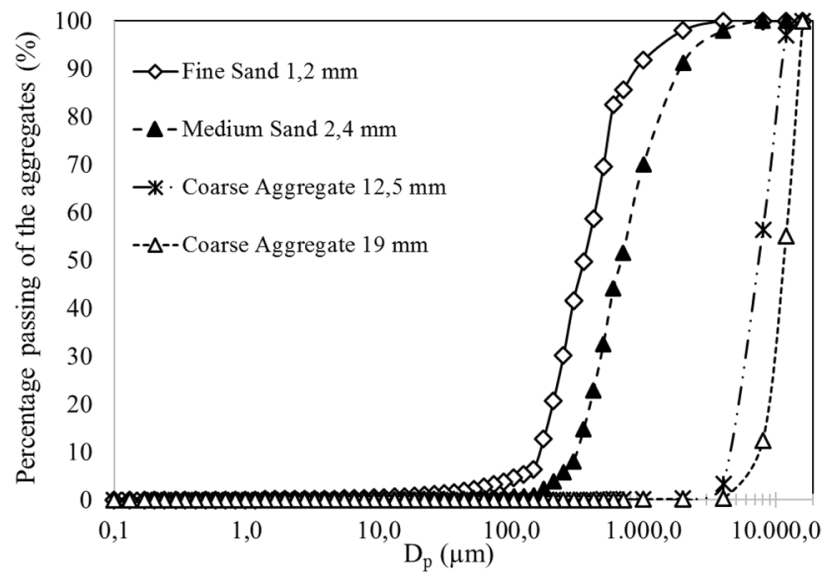

Fig. 1 Granulometric curves versus particles size (Dp) of the all aggregates in the aggregate composition

percent of $60 \%$ of coarse aggregate until $40 \%$. The test is repeated for each proportion; (4) the empty cylinder is determined to obtain the exact quantity of combined aggregates; (5) the aggregates are mixed and poured into a cylindrical container (the maximum size of the aggregate is smaller than three times the container diameter) and three times of voids content and unitary mass measurements are obtained; (6) after the four binary, the test continues with the ternary and quaternary composition, obtained from a binary composition, with other small size aggregate; (7) the results of unitary mass and voids content of each aggregate composition are plotted in a graph, representing unitary mass versus voids content, according to Dìaz [6].

The voids content (e) is determined by Eq. (1) [6], and the porosity (ne), by Eq. (2) [28].

$e=\frac{M_{e m}-M_{u}}{M_{e m}} \times 100$

$\eta_{e}=\frac{V_{v}}{V_{t}}=\frac{V_{t}-V_{s}}{V_{t}}=\frac{e}{1+e}$

where $\eta_{e}$ is the porosity in percentage and e is the voids content, cited as given by Eq. (3):

$e=\frac{V_{v}}{V_{s}}$

$M_{u}$ : unitary mass of the mixture; $M_{e m}$ : specific mass of the mixture, Eq. (4), cited as:

$M_{e m}=\frac{M_{e A_{1}} \times \% A_{1}+M_{e A_{2}} \times \% A_{2}+\cdots+M_{e A_{t}} \times \% A_{t}}{100}$

$\mathrm{V} v$ : voids volume; $\mathrm{V}_{t}$ : total volume; $\mathrm{V}_{s}$ : volume of solid particles composing the aggregate composition; $t$ : the number of aggregates in the mixture $\left(A_{1}, A_{2}, \ldots, A_{t}\right) ; M_{e A t}:$ specific mass of the aggregate.

Therefore, to obtain the packing through the Eq. (5) [29], in percentage, with the experimental porosity $\left(\eta_{\mathrm{e}}\right)$, obtained through the Eq. (2), the packing factor $\left(\mathrm{P}_{\mathrm{f}}\right)$, loose state, is calculate through the Eq. (5. This parameter serves to validate the numerical and experimental data of the aggregates compositions so that it can show the effectiveness of the numerical tool (SSI).

$P_{f}=100-\eta e$

\section{Particle size generation}

This section presents the methodology of each stage used for particle size generation. This methodology is based on technique proposed by Frery et al. [25], which is revised to reproduce the problem of aggregate composition.

\subsection{Granulometric curve and porosity}

A granulometric curve measures the percentage of the sample that falls into pre-established ranges of the grain aggregate sizes. This information is obtained by sieving analyses, for instance, as described in Sect. 2. The results of such measures are provided in tabular form by the cumulative proportion of grains associated for each diameter considered.

The porosity $\eta$ (into interval $[0,1]$ ) is the ratio between the empty spaces in the sample to the total sample volume. It is estimated through the determination of the unitary mass.

The granulometric curve, the proportion of each aggregate composition, the specific mass of dry aggregates and the porosity are the only required input for the rest of the methodology.

\subsection{Number of particles}

This section derives an initial guess for the number of particles to be generated in order to have a good approximation of the desired porosity.

Assume the closed area of interest W has size $\mu(W)$, which must be filled with particles whose radii follow a granulometry $D(\theta)$ defined as presented in Sect. 2. The desired porosity $\eta_{n}$ is also estimated by Eq. (6), from the aggregate sample:

$\eta_{n}=\frac{V_{e}}{\mu(W)}=\frac{\mu(W)-V_{p}}{\mu(W)}$

where $V_{e}$ and $V_{p}$ are the empty and particles areas, respectively. The area of a disk with radius $R$, distributed 
according to the law $D(\theta)$ is a random variable given by $V=\pi R^{2}$. Then, assuming that $N$ particles are placed within W, the Eq. (7) results

$$
V_{p}=\pi \sum_{i=1}^{N} R_{i}^{2}
$$

Using expected values, the Eq. (8) calculate

$$
\begin{aligned}
E\left(V_{p}\right) & =\pi \sum_{i=1}^{N} E\left(R_{i}^{2}\right) \\
& =\pi \sum_{i=1}^{N}\left[\operatorname{Var}\left(R_{i}\right)+E^{2}\left(R_{i}\right)\right] \\
& =N \pi\left[\operatorname{Var}\left(R_{i}\right)+E^{2}\left(R_{i}\right)\right]
\end{aligned}
$$

and both variance (Var) and expected value $(E)$ are given explicitly as functions of the estimated parameter $\theta$, so, the number of particles is given by:

$N=\frac{(1-\eta) \mu(W)}{\pi\left[\widehat{\operatorname{Var}}\left(R_{i}\right)+\hat{E}^{2}\left(R_{i}\right)\right]}$

This number of particles $\mathrm{N}$ is intended as a rough estimate of the needed number of radii required with the desired porosity and granulometry.

\subsection{Generation of particles}

The following procedure presents a sequential approach for obtaining a good approximation to the desired porosity $\eta$, while keeping the statistical properties of the particles. In this procedure, $\mathrm{K}>1$ is a factor which controls the number of extra particles to be generated; we set $K=10$, a good compromise between the relatively low cost of generating outcomes versus the high cost of initializing the generator.

The input data of procedure are the region of interest $\mathrm{W}$, the number of particles $N$, the porosity $\eta$, the granulometric distribution $D(\theta)$ and the factor K. Initially is obtained $r=\left(r_{1}, \ldots, r_{K N}\right)$ samples from independent identically distributed random variables following the $D(\theta)$ granulometric distribution. While the current porosity is greater than the desired porosity, the simple sequential inhibition (SSI) point process (detailed in the following section) is applied for each particle size. The procedure is also finished if it is not possible to insert a new particle in the region of interest. Otherwise, the current porosity is updated according equation below and a new iteration is performed Eq. (10):

$\eta_{i}=\eta_{i}-\frac{\pi r_{i}^{2}}{\mu(W)}$.
If the procedure is successful, the coordinates and radii of the particles inserted in the domain are available, besides the porosity achieved. This procedure places a number of particles obeying the $D(\theta)$ granulometric distribution that approximates the desired porosity $\eta$ on the region of interest $\mathrm{W}$. It is noteworthy that the presented methodology can be immediately extended to compact volumes.

\subsection{Spatial distribution by the SSI process}

The strategy detailed above requires another specific procedure, which implements the sampling from the simple sequential inhibition point processes. Such processes are defined in terms of a region of interest $W$, the number of points and the exclusion radii among them; in this work, the number of points is, instead of specified a priori, controlled by the desired porosity $n$ on $\mathrm{W}$.

The simple sequential inhibition (SSI) point process for $n$ points on $W$ with exclusion radii $r=\left(r_{1}, \ldots, r_{n}\right)$ and maximum number of iterations $j_{\max }$ tries to place $n$ non-overlapping disks of radii $r$ by testing sequentially each disk against the previous ones. The first disk is placed uniformly on W, provided it does not surpass the boundaries. Subsequent steps sample a point in W uniformly and independently of previous disks and verify if there is no overlapping; if there is not, the disk is placed, otherwise up to $j_{\max }$ independent trials are made. The procedure stops when all the disks have been placed, or when the maximum number of iterations has been reached, whichever takes place first.

Notice that the order in which the particles are places is not altered. In particular, if particle $i \geq 2$ does not fit after $\mathrm{j}_{\max }$ attempts, the procedure returns the current state which consists of the previous $\mathrm{i}-1$ particles. Even if particle $i+1$ fits trivially the current state, it is not included. Selecting particles by their size would imply discarding the independent identically distributed random variables model and switching to a collection of correlated deviates: the order statistics.

Dense samples are, typically, harder to obtain than situations of high porosity. In those cases, it is likely that the denser the desired outcome the more results will have to be discarded until a sample with the desired property is obtained.

\section{Results and discussion}

This section presents results related to application of the stochastic point process technique in the analysis of aggregate compositions for concrete from parameters determined experimentally. The example used to illustrate this work is based on samples of aggregates compositions 
for self-compacting concrete studied experimentally by Monteiro et al. [30], in order to study their granulometric properties.

\subsection{Input data}

As mentioned earlier, the input data used to apply the stochastic point process technique in the analysis of aggregate compositions are experimental porosity (ne), granulometry of aggregates, composition (proportion) of the aggregates, specific mass of dry aggregates, and the domain of model. The aggregates compositions are used as input data, as shown in Table 1. This table shows the aggregates composition and their physical proprieties obtain according to Sect. 2.

The packing factor parameter is used as validation between the numerical and experimental data obtained from the SSI application. It should be considered that the packing factor is originated from the parameters (e and ne) obtained by experimental method of determination of maximum Mu. This method inherently takes into consideration factors such as shape and distribution of aggregates. Other effects are also considered, like the wall effect, which is a concern in the selection of the container dimensions used in test of aggregates packing $[6,7]$. While studying the sphericity and roundness of aggregates and their influence on the compressive strength of the concrete, Díaz [5] argues that the form of the grains aggregates should be seen as a factor of great relevance due its intervening not only in the generation of economy of production of concrete, as well as the influence of the rheological properties and the hardened concrete.

\subsection{Output data}

The use of the stochastic point process technique in the analysis of aggregate compositions generates particles without overlap, disperse and loose, i.e., which are not under effect of forces, for example, gravitational forces (Fig. 2). As output data, the use of this technique provides several information, such as virtual images of each aggregate compositions in form of spherical particles in the $2 D$ (disks), numerical porosity ( $\eta$ ), aggregate compositions (\%), number of particles generated (N) and packing factor (Pf). These properties allow to determine some statistical representations, such as histograms and distribution of particles frequency $(\mathrm{Fq})$, in percentage, in function of the particles diameters (Dp), in millimeters. Figures $3,4,5,6$, $7,8,9,10$ and 11 illustrate the virtual images associated to the several cases studied, as well as the proprieties provided by Stochastic SSI and the histograms of frequency distribution. Table 2 shows the comparing between these

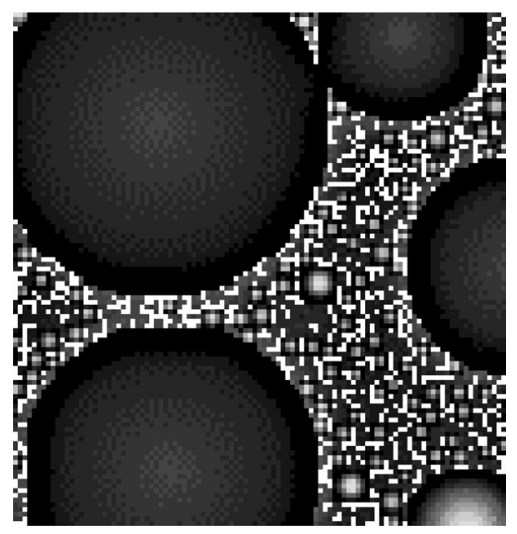

Fig. 2 Representation of dispersion of particles without overlap
Table 1 Composition and proprieties of aggregates, in mass

\begin{tabular}{|c|c|c|c|c|c|c|c|}
\hline \multirow[t]{3}{*}{ Composition } & \multicolumn{4}{|c|}{ Aggregates (\%) } & \multicolumn{3}{|c|}{ Physical Proprieties } \\
\hline & \multicolumn{2}{|c|}{ Fine aggregate } & \multicolumn{2}{|c|}{$\overline{\text { Coarse aggregate }}$} & \multirow[t]{2}{*}{$e(\%)$} & \multirow[t]{2}{*}{$\eta_{e}(\%)$} & \multirow[t]{2}{*}{$\mathrm{Mu}\left(\mathrm{kg} / \mathrm{m}^{3}\right)$} \\
\hline & $\mathrm{F}$ & $M$ & G12 & G19 & & & \\
\hline CFG12 & 45.0 & 0 & 55.0 & 0 & 30.1 & 23.14 & 1866.3 \\
\hline CFG19 & 45.0 & 0 & 0 & 55.0 & 27.6 & 21.63 & 1917.0 \\
\hline CMG12 & 0 & 50.0 & 50.0 & 0 & 34.7 & 25.76 & 1743.5 \\
\hline CMG19 & 0 & 45.0 & 0 & 55.0 & 31.3 & 23.84 & 1822.4 \\
\hline CFMG12 & 23.0 & 27.0 & 50.0 & 0 & 33.6 & 25.15 & 1767.7 \\
\hline CFMG19 & 23.0 & 27.0 & 0 & 50.0 & 30.9 & 23.61 & 1822.9 \\
\hline CFG12G19 & 45.0 & 0 & 25.0 & 30.0 & 31.9 & 24.18 & 1804.5 \\
\hline CMG12G19 & 0 & 40.0 & 27.0 & 33.0 & 32.6 & 24.59 & 1796.3 \\
\hline CFMG12G19 & 22.0 & 28.0 & 22.0 & 28.0 & 32.1 & 24.30 & 1803.4 \\
\hline
\end{tabular}

$C$ composition, $F$ fine sand, $M$ medium sand, G12 gravel $12.5 \mathrm{~mm}, G 19$ gravel $19.0 \mathrm{~mm}$, e voids content, $\eta_{e}$ porosity, Mu unitary mass 

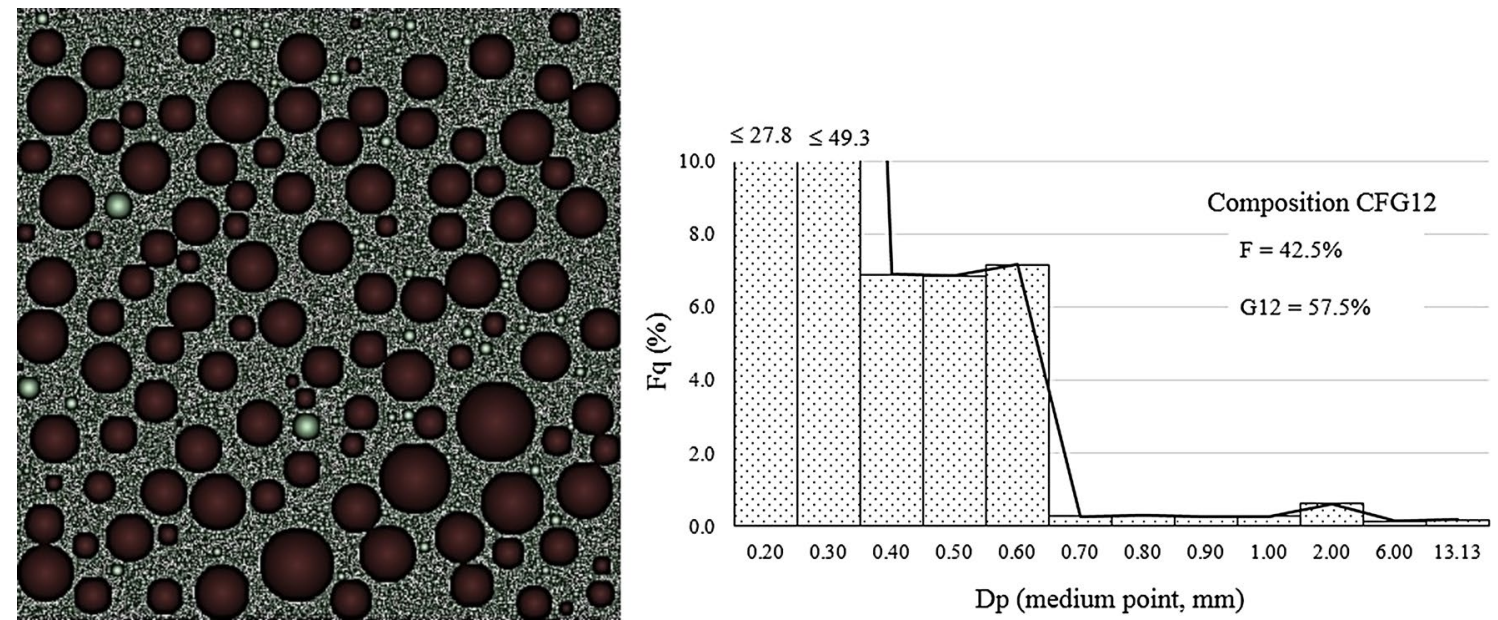

Fig. 3 Virtual image and proprieties provided by Stochastic SSI and histogram of distribution of frequency of $C F G 12: N=41.691 ; P_{f}=76.86 \%$
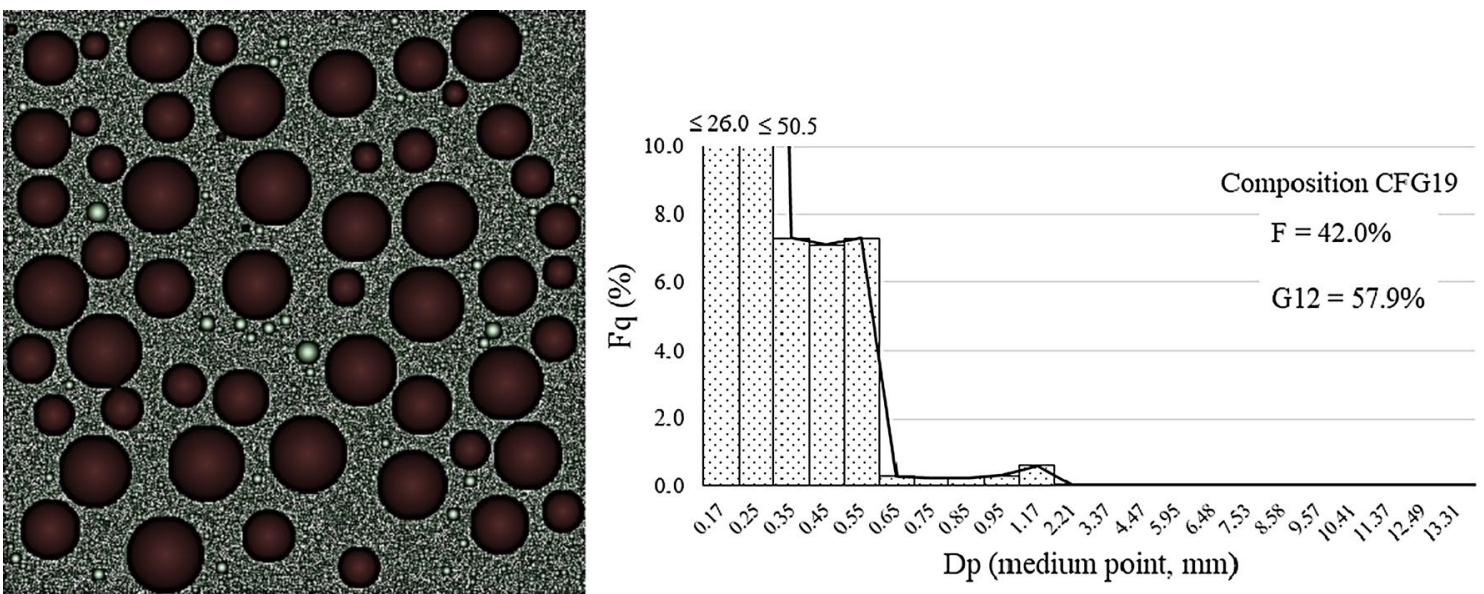

Dp (medium point, $\mathrm{mm}$ )

Fig. 4 Virtual image and proprieties provided by Stochastic SSI and histogram of distribution of frequency of CFG19: $N=51.120 ; P_{f}=78.37 \%$
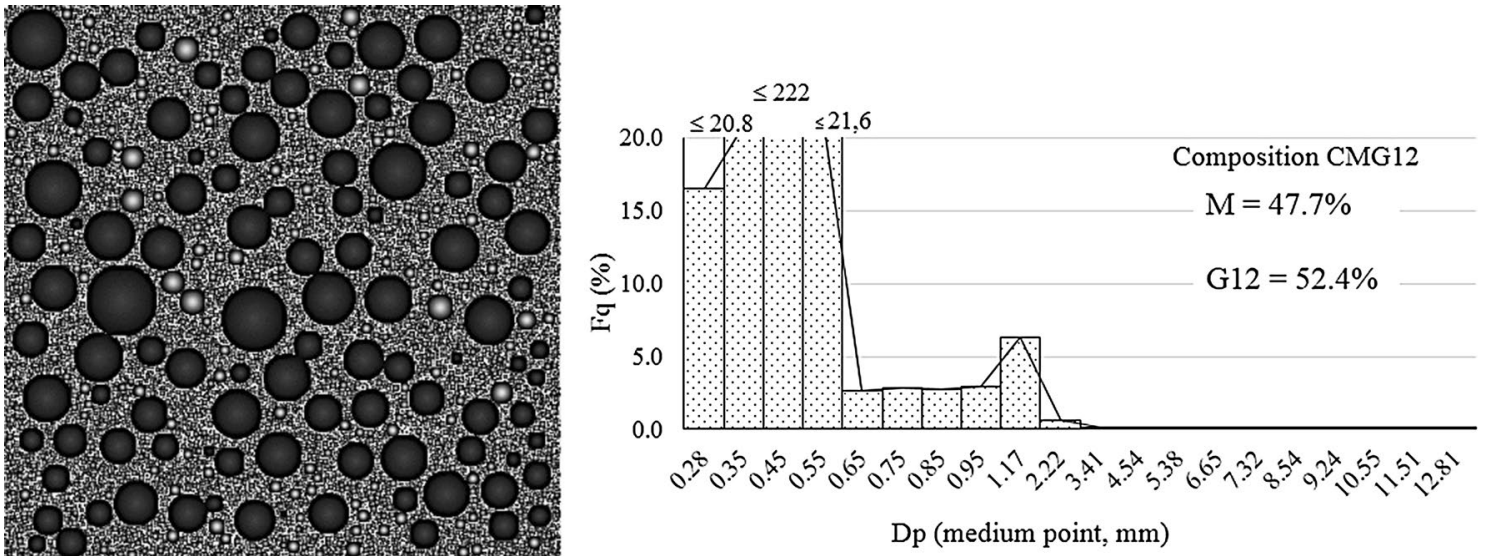

Fig. 5 Virtual image and proprieties provided by Stochastic SSI and histogram of distribution of frequency of $C M G 12: N=12.955 ; P_{f}=74.24 \%$ 

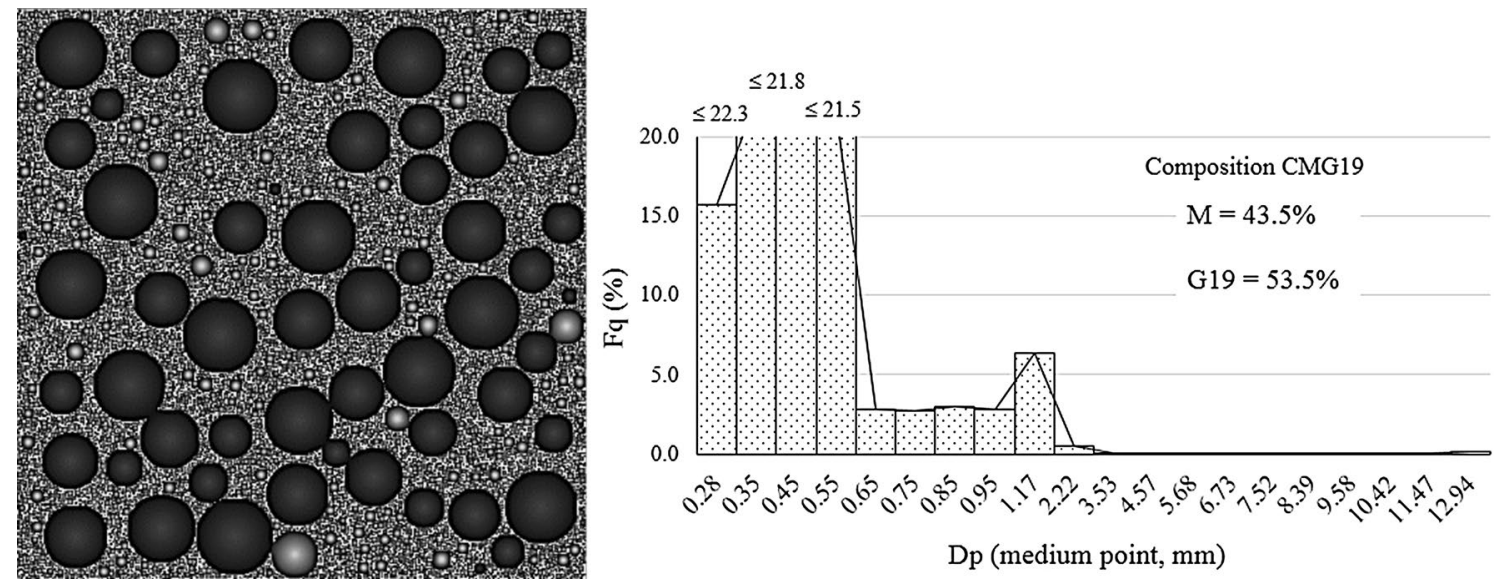

Fig. 6 Virtual image and proprieties provided by Stochastic SSI and histogram of distribution of frequency of CMG19: N=11.822; $P_{f}=76.16 \%$
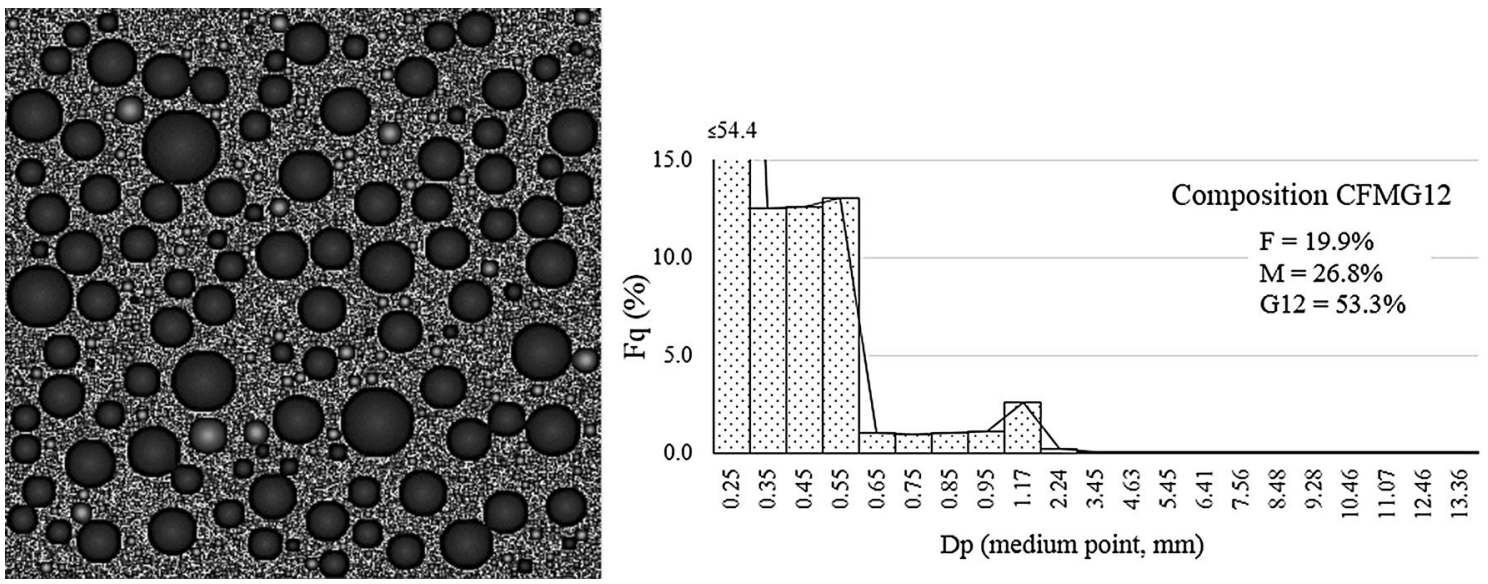

Fig. 7 Virtual image and proprieties provided by Stochastic SSI and histogram of distribution of frequency of CFMG12: N=23.168; $P_{f}=74.85 \%$
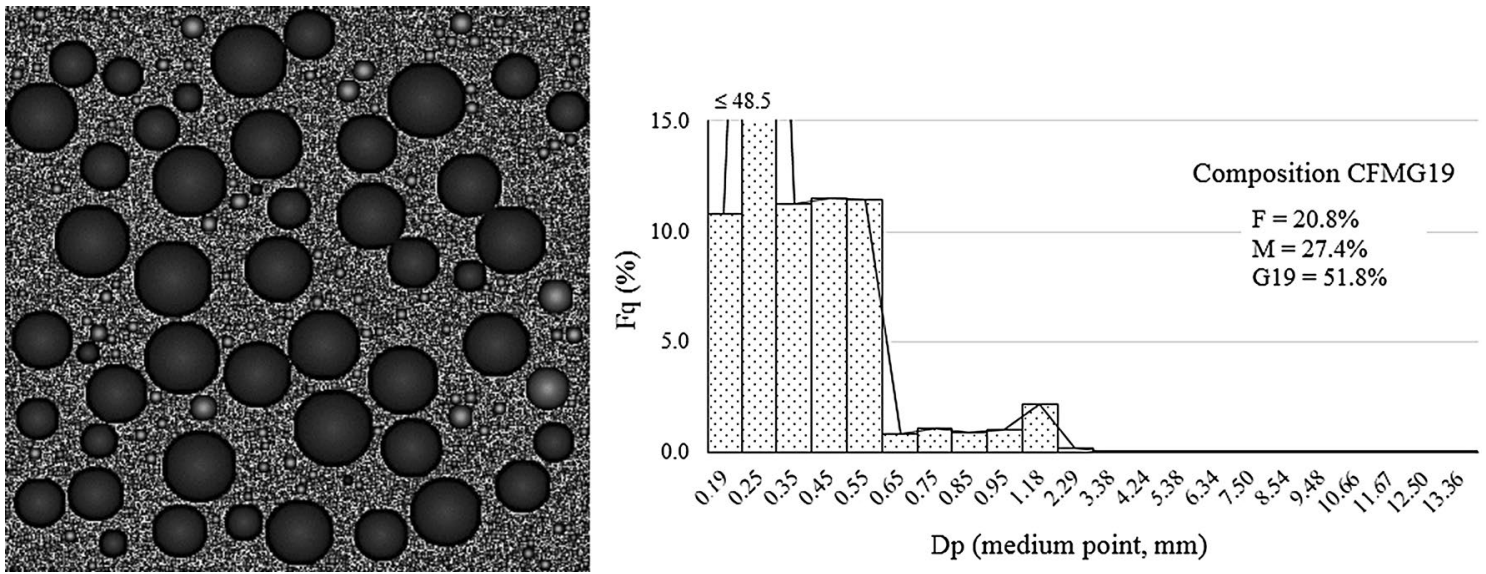

Fig. 8 Virtual image and proprieties provided by Stochastic SSI and histogram of distribution of frequency of CFMG19: N=26.396; $P_{f}=76.39 \%$

\section{SN Applied Sciences}



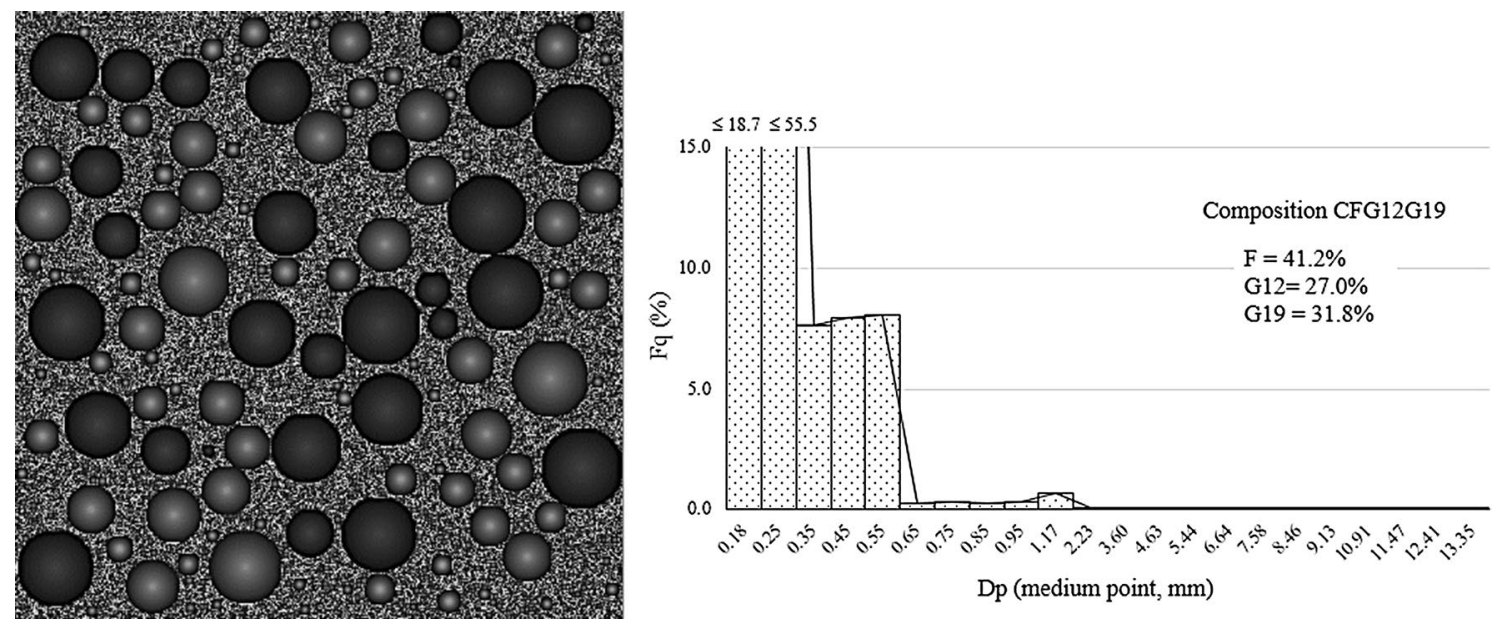

Fig. 9 Virtual image and proprieties provided by Stochastic SSI and histogram of distribution of frequency of CFG12G19: N=36.210; $P_{f}=75.82 \%$
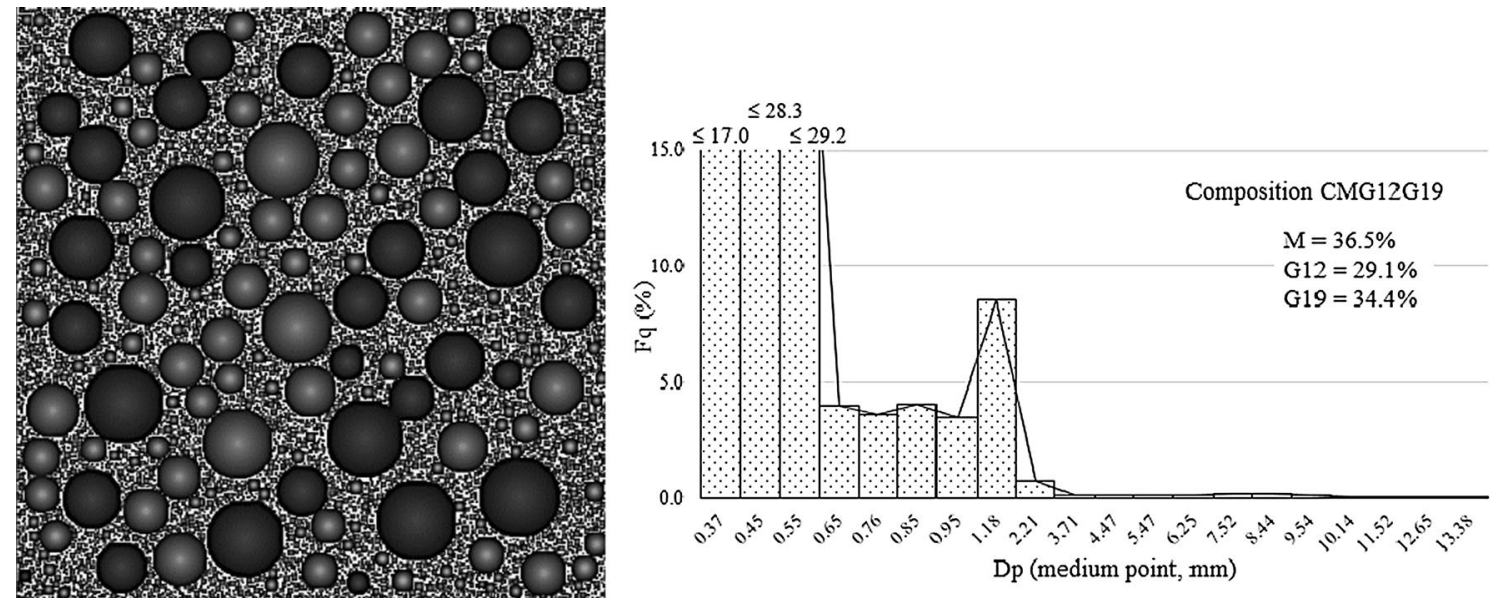

Fig. 10 Virtual image and proprieties provided by Stochastic SSI and histogram of distribution of frequency: CMG12G19: N=7.866; $P_{f}=75.41 \%$
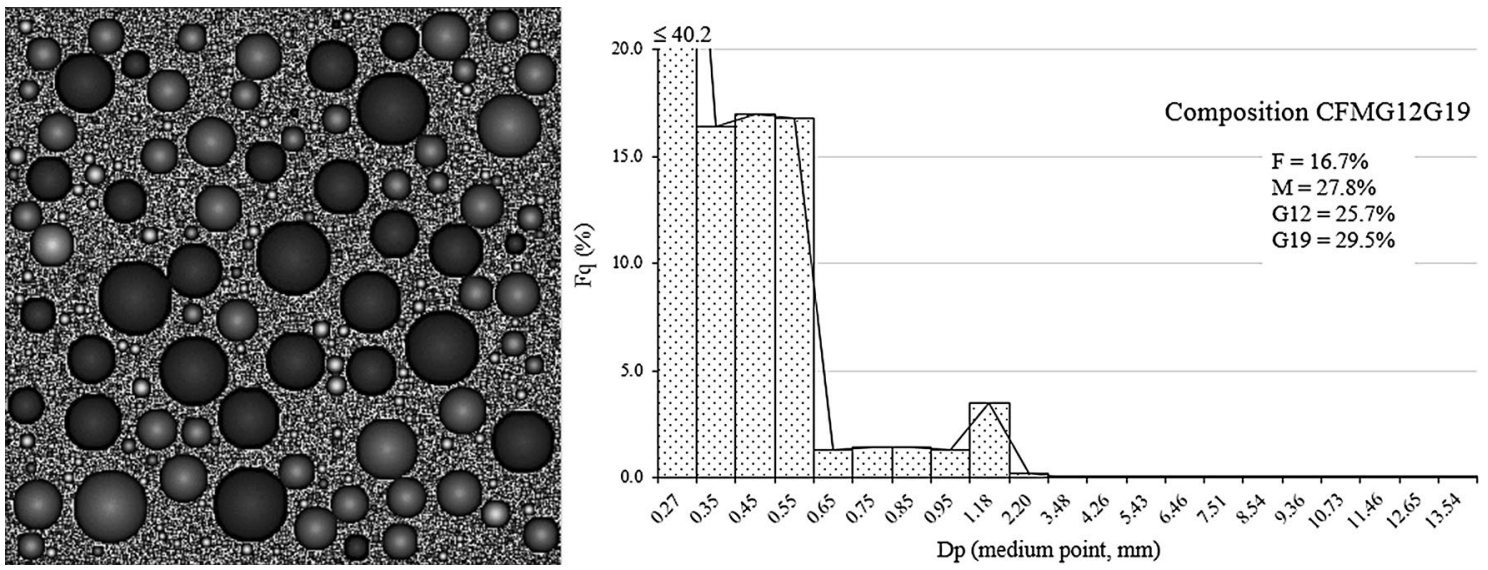

Fig. 11 Virtual image and proprieties provided by Stochastic SSI and histogram of distribution of frequency of CFMG12G19: $\mathrm{N}_{\mathrm{g}}=17,862$; $P_{f}=75.7 \%$ 
Table 2 Experimental and numerical comparison of the characteristics of compositions obtained in the Stochastic SSI

\begin{tabular}{|c|c|c|c|c|c|c|c|c|c|c|}
\hline \multirow[t]{4}{*}{ Compositions } & \multicolumn{8}{|c|}{ Aggregate (\%) } & \multirow[t]{4}{*}{$\eta_{e}(\%)$} & \multirow[t]{4}{*}{$\eta_{n}(\%)$} \\
\hline & \multicolumn{4}{|c|}{ Fine aggregate } & \multicolumn{4}{|c|}{ Coarse aggregate } & & \\
\hline & \multicolumn{2}{|l|}{$\mathrm{F}$} & \multicolumn{2}{|l|}{ M } & \multicolumn{2}{|l|}{$\mathrm{G} 12$} & \multicolumn{2}{|l|}{ G19 } & & \\
\hline & Exp. & Num. & Exp. & Num. & Exp. & Num. & Exp. & Num. & & \\
\hline CFG12 & 45.0 & 42.5 & - & - & 55.0 & 57.5 & - & - & 23.14 & 23.14 \\
\hline CFG19 & 45.0 & 42.0 & - & - & - & - & 55.0 & 57.9 & 21.63 & 21.63 \\
\hline CMG12 & - & - & 50.0 & 47.7 & 50.0 & 52.4 & - & - & 25.76 & 25.76 \\
\hline CMG19 & - & - & 45.0 & 43.5 & - & - & 55.0 & 53.5 & 23.84 & 23.84 \\
\hline CFMG12 & 23.0 & 19.9 & 27.0 & 26.8 & 50.0 & 53.3 & - & - & 25.15 & 25.15 \\
\hline CFMG19 & 23.0 & 20.8 & 27.0 & 27.4 & - & - & 50.0 & 51.8 & 23.61 & 23.61 \\
\hline CFG12G19 & 45.0 & 41.2 & - & - & 25.0 & 27 & 33.0 & 31.8 & 24.18 & 24.18 \\
\hline CMG12G19 & - & - & 40.0 & 36.5 & 27.0 & 29.1 & 33.0 & 34.4 & 24.59 & 24.59 \\
\hline CFMG12G19 & 22.0 & 16.7 & 28.0 & 27.8 & 22.0 & 25.7 & 28.0 & 29.5 & 24.30 & 24.30 \\
\hline
\end{tabular}

$\eta_{e}$ experimental porosity, $\eta_{n}$ numerical porosity achieved numerical information with SSI and the experimental input data.

The use of stochastic point process technique meets the objective of this work which is to obtain an aggregate spatial distribution according to established parameters. To study problems where calculations of interaction forces are required, it is necessary to simulate numerically the set of generated particles, ensuring the equilibrium of the model. To illustrate its use, e.g., two applications can be considered: i) the study of cases involving dry aggregates need to consider gravitational forces, so there will be interaction between the grains, generating surface forces that will take to global balance; ii) the study of cases involving aggregates in suspension the voids, where should be occupied by cement paste. In this case, there will be interaction not only between grains, but also between grains and paste, generating surface forces that will also take to global balance.

The aggregate compositions obtained experimentally have differentiated granular structures, as well as the ones showed in SSI, Figs. 3, 4, 5, 6, 7, 8, 9, 10 and 11. The images help in the visualization of how the particles are distributed, noting the different sizes and the predominance of the grains, enabling to realize, in some of the aggregate compositions, that there is a greater predominance of small particles distributed between the larger particles. i.e., small particles define the porosity of the aggregate compositions, while the thicker ones are distributed between the smallest. Of the results shown in Table 2 , it is evident that the porosity is reduced with the insertion of fine sand (F) among the voids the aggregates G12 and G19.

According to the results of the percentages of the compositions, it is noted that the SSI technique proved to be efficient in the generation of particles with variable sizes in $2 \mathrm{D}$, presenting themselves close to the compositions obtained in the physical experiment. The numerical results of percentages of small aggregates distanced a bit more of the experimental, resulting from the own SSI that interprets the percentages of grains retained on each sieve and presents the diameters of the size of the opening of that sieve, respecting the experimental data (input data). Thus, the SSI technique executes the putting of particles by size, by starting for coarser particles and ending with smaller particles, influencing in the final result of percentage of particles of each composition. For example, these analyzes allow to view the absence of grains in some of the granulometric particles of the compositions CFG12 and CFG19, as well as the concentration of large particles $55 \%$ in both.

In the Figs. 3, 4, 5, 6, 7, 8, 9, 10 and 11 , it is possible to see small white dots, where there is an unfilled space between solid particles. However, there is no difference between $\eta_{\mathrm{e}}$ and $\eta$, Table 2 , thus $\eta$ it is adjusted to obey the granulometry of the aggregate composition given. The factor of experimental packing remains also the same, ranging from 74.24 to $78.37 \%$.

Although there is no differentiation among the experimental and numerical porosities and packing factor, it is realized that there is a significant difference in $\mathrm{N}$ to fit into the $\eta$. The CMG12G19 composition presents the smallest $\mathrm{N}, 15.4 \%$ smaller than the composition CFG19 of higher $\mathrm{N}$. Those that contain only fine sand $(F)$, as small aggregate, CFG12, CFG19 and CFG12G19, in Table 2 are the ones that showed higher $\mathrm{N}$, more than 41 thousand, 36 thousand and 51 thousand particles, respectively.

Figures 3,4 , and 9 , confirming the higher predominance particles of fine sand. The composition, CMG19,

Figure 6 , obtained a lower number of $\mathrm{N}$, due to filling of voids among the large grains of aggregates $\mathrm{G} 19$ by intermediate sand $(M)$, which has less surface area compared to fine sand $(F)$. Containing intermediate sand, the granular 
systems CFMG12, Fig. 7, and CFMG19, Fig. 8, showed a lower number of $\mathrm{N}$, due to smaller proportions of sands $\mathrm{F}$ and $\mathrm{M}$. Even containing both types of large aggregate and a type of sand in ternary compositions CFG12G19 and CMG12G19, Table 2, the difference highlighted among them is that the later obtained a lower number of $\mathrm{N}$, due to the presence of the sand and its smaller proportion (40\%). In the quaternary composition Fig. 11, it is noted that the value of $\mathrm{N}$ is counterbalanced by the proportions of the four types of aggregate, being this quantity sufficient for the SSI technique to adjust the experimental porosity provided.

The histograms corresponding to each aggregate composition show the frequency of particles, in accordance with the diameters provided by the SSI technique, respecting the proportion of aggregates adjusted to achieve the numerical porosity ( $\eta$ ). It is observed in the figures the higher frequency of smaller diameters for the small fractions, which proves that the SSI complies with the ordering of the placement of the sizes of particles (first, the largest ones are inserted, and then the smallest particles) in the domain of $10 \mathrm{~cm} \times 10 \mathrm{~cm}$. The predominance of small particles, equivalent to the aggregates $F$ and $M$, especially, is documented in literature, when it is intended to make voids among the large aggregates be filled in, as far as possible, by the small aggregates, and so forth [9]. From the analysis of the frequency of distributions, it is observed that they are all decreasing asymmetrical polymodal.

The properties of concrete are significantly affected by the aggregate compositions, such as, porosity, density, durability, strength, workability, among others. As an example, high-performance concrete (HPC) can have its compressive strength decreased with the increase of the maximum size of the aggregate, being admitted a low water-cement and higher content of cement, because of the little resistant connection among the coarse and small particles [29]. In addition, when the diameter of the particle tends to increase, the greater is the effect of the coefficient of friction. This parameter, due to its relevance in the behavior of the accommodation of grains in granular structure, is one of the points to be incremented, being treated in future articles.

It is then highlighted the need for a study of aggregates compositions that can balance the coarse and small particles quantity so that it is possible to obtain concretes with better performance.

\subsection{SSI Assessment}

The SSI technique allowed to better understand the influence of different compositions in the determination of percentage of voids, stressing the importance of the selection of sizes and the quantities of particles generated in images. From this understanding, the use of SSI favored the identification of the aggregate composition's modalities (binary, ternary and quaternary), as well as the quantification of the best filling of the voids, fulfilling the input data.

The algorithm used in SSI complied with the physical characteristics entered as input data, which allowed to register, in a granular system of spherical particles, the differentiation of the non-overlapped number of particles and the approximation with the experimental data, which contributes to better representation of different aggregates compositions. The efficiency becomes even better in the requirement of the algorithm in considering the experimental porosity of each composition.

Regarding the output data, the SSI application its shown efficiency in the granulometric generation of particles for the use in concrete, since it is a random generation technique, geometrical and sequential, which obeys the input data. However, the data provided by the SSI require better adjustment so it is possible to interpret other physical parameters also of great influence on the determination of aggregates compositions such as the form of grains, packing under the gravitational effect, coefficient of friction among the grains, wall effect, among other calibrations of this tool for validating experimental trials.

\section{Conclusions}

The results of this study show that:

- The Stochastic SSI is an alternative tool that can be applied in the study of concrete, in view of the information obtained in the study of aggregate compositions. The SSI technique applied responds satisfactorily to adjustment of input data, in addition to allowing to view the arrangement of composition particles, although they are not provided. Also, the technique provides as output data the proportions of aggregates of granular structure and porosity of each composition. It allows to check that the type of aggregate and, consequently, the proportions of these materials interfere in the test for the determination of higher unitary mass and lower volume of voids.

- The adjustments of initial data allowed to validate that the nine aggregates compositions and their respective granulometric distributions interfere with the quantification of the porosity of the aggregate compositions, requiring greater attention in the definition of the selection of granulometric ranges and appropriate proportions of grains that will compose the granular structure. 
- The presence of two kinds of sand, fine and intermediate, filling the spaces between the aggregates in all the compositions, promoted distributions with greater fulfilment of granulometric classes, unlike the compositions where there was only fine sand involving them.

- The generation of the granular structure by applying the SSI serves as a model for simulation studies in which Discrete Element Method (DEM) is effectively applied in the study of the accommodation of the particles. The accommodation of spherical particles through computational simulation, as well as the evaluation of mixtures of concretes with the aggregate compositions discussed in this work, will be treated in future works.

- With the quantities of particles generated by algorithmic, this computational tool contributes not only to the generation of particles, but also in the elaboration of graphs of frequency distributions, allowing to appoint the arrangements of compositions as decreasing asymmetrical polymodal.

- In the light of the output data, specifically of the granulometric distributions studied, the SSI is expected to return the composition and porosity of the aggregate compositions, due to the efficiency by providing the adjusted output data. Thus, it is expected that the dosage of the concrete becomes effective in order to obtain aggregate compositions with lower contents of voids, being reduced thus, the paste content and, consequently, the cement consumption.

Acknowledgements The authors are grateful to CAPES (Coordenação de Aperfeiçoamento de Pessoal de Nível Superior) and CNPq (Conselho Nacional de Desenvolvimento Científico e Tecnológico), FAPEAL (Fundação de Amparo à Pesquisa do Estado de Alagoas), and PETROBRAS S.A. The authors are also thankful to the projects Universal, process number 483935/2012-3, and Casadinho/PROCAD, process number 552300/2011-0, and to the group MECOEFICON/UFAL.

\section{Compliance with ethical standards}

Conflict of interest The authors declare that they have no conflict of interest.

\section{References}

1. Hunger M, Brouwers HJH (2009) Flow analysis of water-power mixtures: application to specific surface area and shape factor. Cement Concr Compos 31(1):39-59

2. Stroeven P, He H, Guo Z, Stroeven M (2009) Particle packing in a model concrete at different levels of the microstructure: evidence of an intrinsic patchy nature. Mater Charact 60(10):1088-1092

3. Fennis AAM, Walraven JC (2012) Using particle packing technology for sustainable concrete mixture design. Heron 57(2):73-101

\section{SN Applied Sciences}

4. Mostofinejad D, Reisi M (2012) A new DEM-based method to predict packing density of coarse aggregates considering their grading and shapes. Constr Build Mater 35:414-420

5. Bolivar OG (2004) Diseno de mezclas de hormigón: métodos empíricos y analíticos. Universidad Nacional de Colombia, Facultad de Minas, Medellín

6. Diaz VO (1998) High performance concrete dosing method. Translation Avelino Aparecido de Pádua, Leonel Tula Sanabria, Nelson Diaz Brito. PINI, São Paulo

7. Nanthagopalan P, Anthanam M (2012) An empirical approach for the optimisation of aggregate combinations for self-compacting concrete. Mater Struct 45(8):1167-1179

8. Goltermann P, Johansen V, Palbol L (1997) Packing of aggregates: an alternative tool to determine the optimal aggregate mix. Mater J 94(5):435-443

9. Mangulkar MN, Jamkar SS (2013) Review of particle packing theories used for concrete mix proportioning. Contrib Pap 4(5):143-148

10. De Larrard F (1999) Concrete optimisation with regard to packing density and rheology. In: 3rd RILEM international symposium on rheology of cement suspensions such as fresh concrete, France

11. Fennis SAAM (2011) Design of ecological concrete by particle packing optimization. http://repository.tudelft.nl/view/ir/ uuid:5a1e445b-36a7-4f27-a89a-d48372d2a45c/

12. He H, Guo Z, Stroeven P, Stroeven M, Sluys LJ (2009) Characterization of the packing of aggregate in concrete by a discrete element approach. Mater Charact 60(10):1082-1087

13. Jamkar SS, Rao CBK (2004) Index of aggregate particle shape and texture of coarse aggregate as a parameter for concrete mix proportioning. Cem Concr Res 34(11):2021-2027

14. Cundall PA, Strack ODL (1979) A discrete numerical method for granular assemblies. Geotechnique 29:47-65

15. Cundall PA (1998) Formulation of a three dimensional distinct element model part I: a scheme to detect and represent contacts in a system of many polyhedral blocks. Int J Rock Mech Min Sci Geomech Abstr 25(3):107-116

16. Zarate F, Rojek J, Oñate E, Miquel J (2001) Modelling of rock, soil and granular materials using spherical elements. In: Z Waszczyszyn, J Pamin (eds) ECCM-2001 CD-ROM proceedings of the second european conference on computational mechanics in solids, structures and coupled problems in engineering, Cracow

17. Zarate F, Rojek J, Oñate E, Miquel J (2004) Thermomechanical discrete element formulation for wear analysis of rock cutting tools. Tech rep CIMNE

18. Fieller NRJ, Flenley EC (1992) Statistics of particle size data. Appl Stat 41(1):127-146

19. Eshel G, Levy GJ, Mingelgrin U, Singer MJ (2004) Critical evaluation of the use of laser diffraction for particle-size distribution analysis. Soil Sci Soc Am J 68(3):736-743

20. Feng YT, Han K, Owen DRJ (2003) Filling domains with disks: an advancing front approach. Methods Eng. 56(5):699-731

21. Cui L, O'Sullivan C (2003) Analysis of a triangulation based approach for specimen generation for discrete element simulation. Granular Matter 5:135-145

22. Barndorf-Nielsen OE (1977) Exponentially decreasing distributions for logarithm of particle-size. Proc R Soc Lond Ser A Math Phys Eng Sci 353:401-419

23. Labra C, Oñate E (2009) High-density sphere packing to discrete element method simulations. Commun Numer Methods Eng 25(7):837-849

24. Lohner $R$, Oñate $E$ (2004) A general advancing front technique for filling space with arbitrary objects. Int. J. Numer. Methods Eng. 61:1977-1991 
25. Frery AC, Rivarola-Duarte L, Ramos VCL, Lira WWM (2012) Stochastic particle packing with specified granulometry and porosity. Granul Matter 14(1):27-36

26. Associação Braileira de Normas Técnicas (2003) NBR NM 248: Agregados-Determinação da composição granulométrica. Rio de Janeiro

27. Associação Braileira de Normas Técnicas (2006) NBR NM 45: Agregados-Determinação da massa unitária e do volume de vazios. Rio de Janeiro

28. Pinto CDS (2002) Basic course in soil mechanics in 16 classes, 2nd edn. In: Texts workshop, São Paulo
29. Oliveira IR, Studart AR, Pileggi RG, Pandolfelli VC (2000) Particle dispersion and packing: principles and applications in ceramic processing. Fazendo Arte Editorial, São Paulo

30. Monteiro RDS, Gomes PCC, Moraes KAM (2015) Aggregates compositions to obtain self-compacting concrete

Publisher's Note Springer Nature remains neutral with regard to jurisdictional claims in published maps and institutional affiliations. 